\title{
Traditional Chinese Medicine Improves Activities of Daily Living in Parkinson's Disease
}

\author{
Weidong Pan, ${ }^{1,2}$ Shin Kwak, ${ }^{2}$ Yun Liu, ${ }^{1}$ Yan Sun, ${ }^{1}$ Zhenglong Fang, ${ }^{1}$ Baofeng Qin, ${ }^{1}$ \\ and Yoshiharu Yamamoto ${ }^{3}$ \\ ${ }^{1}$ Department of Neurology, Shuguang Hospital, Shanghai University of TCM, 185, Pu-An Road, Shanghai 200021, China \\ ${ }^{2}$ Department of Neurology, Graduate School of Medicinel, The University of Tokyo, 7-3-1, Hongo, Bunkyo-ku, Tokyo 113-8655, Japan \\ ${ }^{3}$ Educational Physiology Laboratory, Graduate School of Education, The University of Tokyo, 7-3-1, Hongo, Bunkyo-ku, \\ Tokyo 113-0033, Japan
}

Correspondence should be addressed to Weidong Pan, panwd@medmail.com.cn

Received 17 June 2010; Accepted 17 March 2011

Academic Editor: Ray Chaudhuri

Copyright () 2011 Weidong Pan et al. This is an open access article distributed under the Creative Commons Attribution License, which permits unrestricted use, distribution, and reproduction in any medium, provided the original work is properly cited.

\begin{abstract}
We evaluated the effects of a traditional Chinese medicine (TCM), named Zeng-xiao An-shen Zhi-chan 2 (ZAZ2), on patients with Parkinson's disease (PD). Among 115 patients with idiopathic PD enrolled (mean age, $64.7 \pm 10.2$ years old), 110 patients $(\mathrm{M}=$ $65, \mathrm{~F}=45$; mean age, $64.9 \pm 10.7$ years old) completed the study. Patients took either ZAZ2 $(n=59)$ or placebo granule $(n=56)$ in a blind manner for 13 weeks while maintaining other anti-Parkinson medications unchanged. All participants wore a motion logger, and we analyzed the power-law temporal autocorrelation of the motion logger records taken on 3 occasions (before, one week, and 13 weeks after the drug administration). Drug efficacy was evaluated with the conventional Unified Parkinson Disease Rating Scale (UPDRS), as well as the power-law exponent $\alpha$, which corresponds to the level of physical activity of the patients. ZAZ2 but not placebo granule improved the awake-sleep rhythm, the UPDRS Part II, Part II + III, and Part IV scores, and the $\alpha$ values. The results indicate that ZAZ2 improved activities of daily living (ADL) of parkinsonism and, thus, is a potentially suitable drug for long-term use.
\end{abstract}

\section{Introduction}

Conventional anti-parkinsonism drugs effectively ameliorate the symptoms of patients with Parkinson's disease (PD) during the initial several years of onset, but become increasingly less effective and induce motor fluctuations including wearing-off, on-off, dopa-induced dyskinesia, and agonistinduced sleep attack [1-5]. PD patients not infrequently suffer from nonmotor symptoms, such as neuropsychiatric symptoms, autonomic symptoms, gastrointestinal symptoms, sensory symptoms, nonmotor fluctuations (autonomic symptoms, cognitive or psychiatric symptoms, sensory symptoms including pain), fatigue, and sleep disturbance [6-8], and these nonmotor symptoms may be intrinsic to the disease pathology or may be the result of treatment with dopaminergic agents. Several studies have established that the nonmotor symptoms of $\mathrm{PD}$ are common, occur across all stages of PD, and are a key determinant of quality of life [7].

Herbal remedies have a long history of use (particularly in East Asian countries) for alleviating various symptoms and have been increasingly used as alternative medicines worldwide, including the United States [9]. Traditional Chinese medicines (TCMs) ameliorate various symptoms, particularly the ageing-related symptoms $[10,11]$, and hence are likely to be beneficial for chronic diseases such as PD [1214]. Good compliance for long-term use with few side effects may be another merit of TCM suitable for patients with PD [12-14].

In order to evaluate the effects of TCM on symptoms of parkinsonism, we used Zeng-xiao An-shen Zhi-chan 2 (ZAZ2) in this study. In addition, we adopted a recently developed method analyzing the power-law temporal autocorrelation of wrist activity measured with a motion logger 
[15-17] in order to evaluate the efficacy of ZAZ2 on parkinsonism. Pan et al. showed that the power-law exponent $(\alpha)$ for higher levels or at the so-called local maxima of coefficients of the wavelet transform significantly and in correlation with the symptom severity of PD patients [15]; hence analysis of $\alpha$ is likely to be useful for evaluating the effects of TCM on parkinsonism as a whole. The aim of the present study was to evaluate the ameliorating effects of ZAZ2 on impaired motor and nonmotor symptoms of PD patients using the UPDRS scores, secondary symptoms scores [18], and also by analyzing the mean activity levels and the power-law temporal exponents of MicroMiniMotionlogger, Ambulatory Monitoring Inc. (AMI) scores.

\section{Methods}

2.1. Subjects. Of the $140 \mathrm{PD}$ patients who visited the clinic at the Department of Neurology of Shuguang Hospital Affiliated to Shanghai University of TCM between July 2008 and April 2010, 115 patients with idiopathic PD (mean age \pm SD, $64.7 \pm 10.2$ years old, mean duration of illness, $5.5 \pm 7.3$ years) who fulfilled the inclusion criteria, were invited to participate in the study. The UK Parkinson's Disease Society Brain Bank clinical diagnostic criteria were used [19]. PD was defined by the presence of at least two of the four cardinal features (bradykinesia, tremor, rigidity, and postural reflex abnormality). Other forms of parkinsonism based on laboratory tests such as MRI were excluded. All the patients were at least 40 years of age and were evaluated in the middle of their levodopa dose cycle at maximal mobility (on) for the severity of parkinsonism, and signed informed consent before participation. Next, the patients were double blindly grouped into the TCM group $(n=59$, $64.27 \pm 11.8)$ or the placebo group $(n=56,63.91 \pm 13.9)$ (Table 1). Patients were randomly assigned to the ZAZ2 or placebo group and given random numbers by a study coordinator, who also encoded the drugs with matching random numbers. Neither the patients nor the researchers monitoring the outcome knew which patient was receiving which treatment, until the study was over and the random code was broken. Anti-parkinsonism drug administration was not changed throughout the experiment. The study was approved by The Ethics Committee of Shuguang Hospital Affiliated to Shanghai University of TCM, and performed under the principles outlined in the Declaration of Helsinki; all subjects provided informed consent in accordance with institutional requirements prior to participation in the study.

2.2. Additional Treatment. Zeng-xiao An-shen Zhi-chan 2 (ZAZ2), the TCM used in this study, is a granule made up of 14 kinds of herbs: Uncaria rhynchophylla $10 \mathrm{~g}$, Rehmanniae radix $10 \mathrm{~g}$, Cornus officinalis $8 \mathrm{~g}$, Asnaragus cochinchinensic $10 \mathrm{~g}$, Paeonia lactiflora $10 \mathrm{~g}$, Desertliving cistanche $10 \mathrm{~g}$, Puerariae radix $10 \mathrm{~g}$, Arisaema consanguineum Schott $10 \mathrm{~g}$, Salviae Miltiorrhizae radix $10 \mathrm{~g}$, Acorus tatarinowii $10 \mathrm{~g}$, Curcuma longa Linn $12 \mathrm{~g}$, Morindae officinalis radix $10 \mathrm{~g}$, Rhizoma gastrodiae $10 \mathrm{~g}$, and Rhizoma chuanxiong $10 \mathrm{~g}$. ZAZ2 is commonly used in "insufficiency of Kidney yang" in China. Placebo granules were made up of 5 kinds of herbs:
TABle 1: Patient characteristics.

\begin{tabular}{lcc}
\hline & Placebo $(n=54)$ & ZAZ2 $(n=56)$ \\
\hline Age (yr) & $63.1 \pm 10.2$ & $62.82 \pm 10.31$ \\
Men/Women & $32 / 22$ & $34 / 22$ \\
Disease duration (yr) & $5.81 \pm 3.24$ & $5.73 \pm 4.81$ \\
Hoehn \& Yahr stages & $2.35 \pm 1.33$ & $2.37 \pm 1.13$ \\
Levodopa/DCI (mg/day) & $396.61 \pm 159.24$ & $390.59 \pm 164.71$ \\
Pramipexole (mg/day) & $1.03 \pm 0.69$ & $1.00 \pm 0.79$ \\
$\begin{array}{l}\text { Selegiline Hydrochloride } \\
\text { (mg/day) }\end{array}$ & $8.97 \pm 5.66$ & $9.28 \pm 4.95$ \\
\hline
\end{tabular}

DCI: decarboxylase inhibitor.

Largehead atractylodes rhizome $10 \mathrm{~g}$, Poria cocos (Schw.) wolf $10 \mathrm{~g}$, Jobstears seed $10 \mathrm{~g}$, Malt $10 \mathrm{~g}$, and Chinese date $10 \mathrm{~g}$. These 5 herbs have no activity in terms of traditional Chinese medicine [13]. Patients were instructed to take one package (8 g) of ZAZ2 or placebo soluble granule three times a day at least $30 \mathrm{~min}$ before or after the ingestion of other drugs for three consecutive months (13 weeks). The shape and color of ZAZ2 and the placebo soluble granule are very alike and cannot be distinguished from one another by appearance or aqueous solution taste. ZAZ2 and the placebo granule were made by the manufacturing laboratory of Shuguang Hospital Affiliated to Shanghai University of TCM. The trial was carried out as a randomized, double-blind, parallel group study.

2.3. Equipment. All patients wore a small watch-type activity monitor equipped with a computer (MicroMiniMotionlogger, Ambulatory Monitoring, Inc, Ardsley, New York) on the wrist of their nondominant hand for seven consecutive days before taking test granule (week 0), one week (week 1), and 13 weeks (week 13) after taking test granule. Zero-crossing counts were recorded every one minute to register and quantify human physical activity [15], and the data was stored in internal memory. After recording, data were transmitted to an external computer by software installed on the device.

2.4. Assessments. Daily profiles and mean counts: We plotted the activity scores for 7 consecutive days to see the daily profiles and biological rhythms of each patient (Figure 1). The records acquired during awake times and sleep times were separated with Action-W, Version 2 (Ambulatory Monitors Inc., Ardsley, NY, USA). The mean counts during awake times and sleep times were separately calculated for each record (Table 2 and Figure 2(a)).

UPDRS Scores. The UPDRS of all patients were evaluated at week 0 , week 1 , and week 13 by neurologists who were blinded to the test granule.

Secondary Symptom Score [18]. This score is conventionally used in China to evaluate the effects of anti-parkinsonism drugs and consists of 8 parts, including the assessments of nonfluent speech, vertigo, insomnia/nightmares, headache, 
TABLE 2: Results of clinical evaluation between before and after test granule administration.

\begin{tabular}{|c|c|c|c|c|c|c|}
\hline & \multicolumn{3}{|c|}{ Placebo $(n=54)$} & \multicolumn{3}{|c|}{$\mathrm{ZAZ}(n=56)$} \\
\hline & Week 0 & Week 1 & Week 13 & Week 0 & Week 1 & Week 13 \\
\hline UPDRS total score & $46.6 \pm 16.3$ & $44.7 \pm 15.3$ & $45.9 \pm 18.1$ & $46.3 \pm 17.1$ & $37.1 \pm 11.2^{* \# \#}$ & $40.7 \pm 15.1^{\text {*\# }}$ \\
\hline UPDRS I & $2.5 \pm 0.7$ & $2.3 \pm 1.1$ & $2.4 \pm 1.2$ & $2.6 \pm 0.8$ & $2.1 \pm 0.7^{*}$ & $2.3 \pm 0.9$ \\
\hline UPDRS II & $15.7 \pm 9.3$ & $14.8 \pm 11.2$ & $15.3 \pm 11.6$ & $15.9 \pm 11.3$ & $12.5 \pm 4.6^{* \#}$ & $13.4 \pm 9.8^{* \#}$ \\
\hline UPDRS III & $25.5 \pm 12.9$ & $23.8 \pm 10.6^{*}$ & $24.9 \pm 12.7$ & $25.4 \pm 10.1$ & $19.3 \pm 9.8^{* \#}$ & $21.6 \pm 10.4^{*}$ \\
\hline UPDRS IV & $3.1 \pm 1.1$ & $2.9 \pm 1.6$ & $3.0 \pm 1.4$ & $3.2 \pm 1.4$ & $2.6 \pm 0.8^{* \#}$ & $2.7 \pm 1.3^{* \#}$ \\
\hline Awake time (counts/min) & $98.5 \pm 14.1$ & $102.6 \pm 18.9$ & $100.7 \pm 16.9$ & $99.8 \pm 17.8$ & $126.7 \pm 13.4^{* \# \#}$ & $118.4 \pm 11.8^{* \# \#}$ \\
\hline Sleep time (counts/min) & $42.9 \pm 17.1$ & $38.8 \pm 15.6^{*}$ & $40.1 \pm 14.8$ & $43.2 \pm 11.6$ & $35.6 \pm 13.6^{* \#}$ & $32.8 \pm 13.6^{* \#}$ \\
\hline$\alpha$ (awake time) & $0.97 \pm 0.21$ & $0.95 \pm 0.28$ & $0.96 \pm 0.18$ & $0.97 \pm 0.24$ & $0.88 \pm 0.21^{* \#}$ & $0.86 \pm 0.19^{* \# \#}$ \\
\hline$\alpha$ (sleep-time $)$ & $1.19 \pm 0.28$ & $1.16 \pm 0.27$ & $1.15 \pm 0.29$ & $1.18 \pm 0.26$ & $1.04 \pm 0.22^{* \#}$ & $1.02 \pm 0.18^{* \# \#}$ \\
\hline
\end{tabular}

Data presented are mean $\pm \mathrm{SD}$. ${ }^{*} P<.05$; ${ }^{* *} P<.01$ compared to week 0 (repeated-measure ANOVAs); ${ }^{\#} \mathrm{P}<.05$; ${ }^{\# \#} P<.01$ compared to placebo (Bonferroni test); UPDRS: Unified Parkinson's Disease Rating Scale; $\alpha$ : power-law exponent.

sweating or night sweats, tiredness, sense of cold, and dysuria. In this study, the secondary symptom scores were evaluated in week 0 , week 1 , and week 13 for all participants by the same neurologists, and it reflects the opinion of PD patients (Table 3).

Power-Law Temporal Analysis $\alpha$. The methods for power-law temporal analyses were the same as those described in Pan et al. [15]. The awake time and sleep time data were used separately for the power-law temporal analyses. After integrating the time series, the data were wavelet-transformed using the third derivative of the Gaussian function as the socalled "mother wavelet." The wavelet coefficients (W (S)) at each point along the time series and at different timescales (S) were obtained by convolving the mother wavelet with the time series. This approach facilitates the probing of transient increases or decreases in detrended activity records at different timescales. The transient increases (low-high-low level activity patterns) yielded local maxima of the wavelet coefficients at their time points, while the decreases (highlow-high level activity patterns) yielded local minima of the wavelet coefficients. Next, the squared wavelet coefficients at the local maxima or minima were averaged for all the data points, and the power-law exponent $(\alpha)$ was obtained separately for local maxima and minima as the slope of a straight line fitted in the double-logarithmic plot of $S$ versus $\mathrm{W}(\mathrm{S})^{2}$ in the range of $\mathrm{S}$ corresponding to 8 to $35 \mathrm{~min}$. The power-law exponent of maxima has been successfully used for the assessment for PD in previous studies $[15,20]$. In this study, we analyzed the local maxima separately for the awake time and sleep time of each record (Table 2 and Figure 2(b)).

For the safety assessments, each patient underwent a physical examination by a physician and laboratory tests for blood counts and biochemistry, and urinalysis at each visit.

2.5. Statistical Analysis. Repeated-measure ANOVAs were conducted to test the differences among week 0 , week 1 , and week 13 in the ZAZ2 and placebo groups. When a significant difference was detected, a post-hoc test (Bonferroni test) was conducted between the ZAZ2 and placebo groups compared for the UPDRS total score, UPDRS Part I, Part II, Part III,
Part II + Part III, and Part IV, mean values, and the powerlaw temporal $\alpha$ in awake time and sleep time. A significant difference was defined as $P<.05$. SPSS windows Version 17.0 was used for statistical analyses. All data are expressed as the mean \pm standard deviation.

\section{Results}

Five patients dropped out of the study; one patient in the ZAZ2 group was unable to tolerate the bitter taste of ZAZ2, while two in the ZAZ2 group and two in the placebo group dropped out due to a conflict with other TCM prescribed for concomitant diseases. Neither physical examination nor laboratory tests revealed any adverse changes after additional treatment in either group.

The post-hoc test revealed no significant differences in baseline (week 0) UPDRS scores, Hoehn \& Yahr stages, mean counts, and power-law temporal exponent $\alpha$ values, or in the dosage of L-dopa/DCI, Dopamine agonist or monoamine oxidase B, between the ZAZ2 and placebo groups (Tables 1 and 2).

Daily profiles of AMI counts clearly demonstrated improvement of the biological rhythm after the additional treatment in the ZAZ2 group (Figure 1(a)) but not in the placebo group (Figure 1(b)). Patients in the ZAZ2 group showed a more frequent switch from high activity to low activity in awake time and lower activity during sleep time after ZAZ2 than before ZAZ2 (Figures 1(a) and 2(a), Table 2, $P<.05$, Bonferroni test). Such changes were not observed after placebo granule intake (Figures 1(b) and 2(a), Table 2).

When the effects of ZAZ2 were evaluated with UPDRS scores, significant and persistent improvements were found in the part II, parts II + III, and part IV scores (Table 2). Although some parts of UPDRS improved in week 1 in both the ZAZ2 and placebo groups, the improvement did not persist until week 13 (repeated-measure ANOVAs Table 2). There were significant differences in UPDRS Part II, Part II + Part III, and Part IV scores at week 13 between the ZAZ2 group and placebo group $(P<.05$, Bonferroni test; Table 2$)$.

The local power-law exponent $\alpha$, given by a slope of the $\log \mathrm{S}$ versus $\log \mathrm{W}(\mathrm{S})^{2}$ relationship, characterizes the nature 
TABLE 3: Effects on secondary symptoms of PD.

\begin{tabular}{cccccccccc}
\hline Group & Time & $\begin{array}{c}\text { Nonfluent } \\
\text { speech }\end{array}$ & Vertigo & $\begin{array}{c}\text { Insomnia/ } \\
\text { nightmare }\end{array}$ & Headache & $\begin{array}{c}\text { Sweating or } \\
\text { night sweats }\end{array}$ & Tiredness & Sense of cold & Dysuria \\
\hline \multirow{2}{*}{ Placebo } & 0 weeks & $1.12 \pm 0.59$ & $1.31 \pm 0.97$ & $2.67 \pm 0.87$ & $1.03 \pm 0.75$ & $2.13 \pm 1.32$ & $1.70 \pm 0.97$ & $1.78 \pm 0.39$ & $2.29 \pm 1.02$ \\
& 1 weeks & $0.69 \pm 0.32^{*}$ & $1.12 \pm 0.69$ & $2.40 \pm 0.69^{*}$ & $0.96 \pm 0.36^{*}$ & $1.87 \pm 0.58$ & $1.35 \pm 0.69^{*}$ & $1.39 \pm 0.81$ & $1.69 \pm 0.92^{*}$ \\
& 13 weeks & $1.02 \pm 0.36$ & $1.28 \pm 0.53$ & $2.45 \pm 0.38$ & $0.99 \pm 0.65$ & $2.18 \pm 0.56$ & $1.58 \pm 0.66$ & $1.64 \pm 0.58$ & $2.18 \pm 1.30$ \\
\hline \multirow{2}{*}{ ZAZ2 } & 0 weeks & $1.08 \pm 0.74$ & $1.33 \pm 0.83$ & $2.77 \pm 0.98$ & $0.92 \pm 0.56$ & $2.11 \pm 0.68$ & $1.66 \pm 0.57$ & $1.90 \pm 0.67$ & $2.23 \pm 0.69$ \\
& 1 weeks & $0.56 \pm 0.28^{*}$ & $0.84 \pm 0.26^{* \#}$ & $2.03 \pm 0.78^{*}$ & $0.64 \pm 0.28^{* \# \#}$ & $1.38 \pm 0.69^{* \#}$ & $1.21 \pm 0.46^{*}$ & $1.48 \pm 0.57^{*}$ & $1.43 \pm 0.31^{* \#}$ \\
& 13 weeks & $0.65 \pm 0.33^{* \# \#}$ & $0.95 \pm 0.37^{* \#}$ & $1.73 \pm 0.38^{* \#}$ & $0.63 \pm 0.19^{* \#}$ & $1.48 \pm 0.28^{* \# \#}$ & $1.27 \pm 0.51^{* \#}$ & $1.58 \pm 0.61$ & $1.46 \pm 0.36^{* \# \#}$ \\
\hline
\end{tabular}

Data presented are mean $\pm \mathrm{SD} ;{ }^{*} P<.05 ;{ }^{* *} P<.01$ compared with 0 weeks (repeated-measure ANOVAs). ${ }^{\#} P<.05$; ${ }^{\# \#} P<.01$ compared to placebo (Bonferroni test).

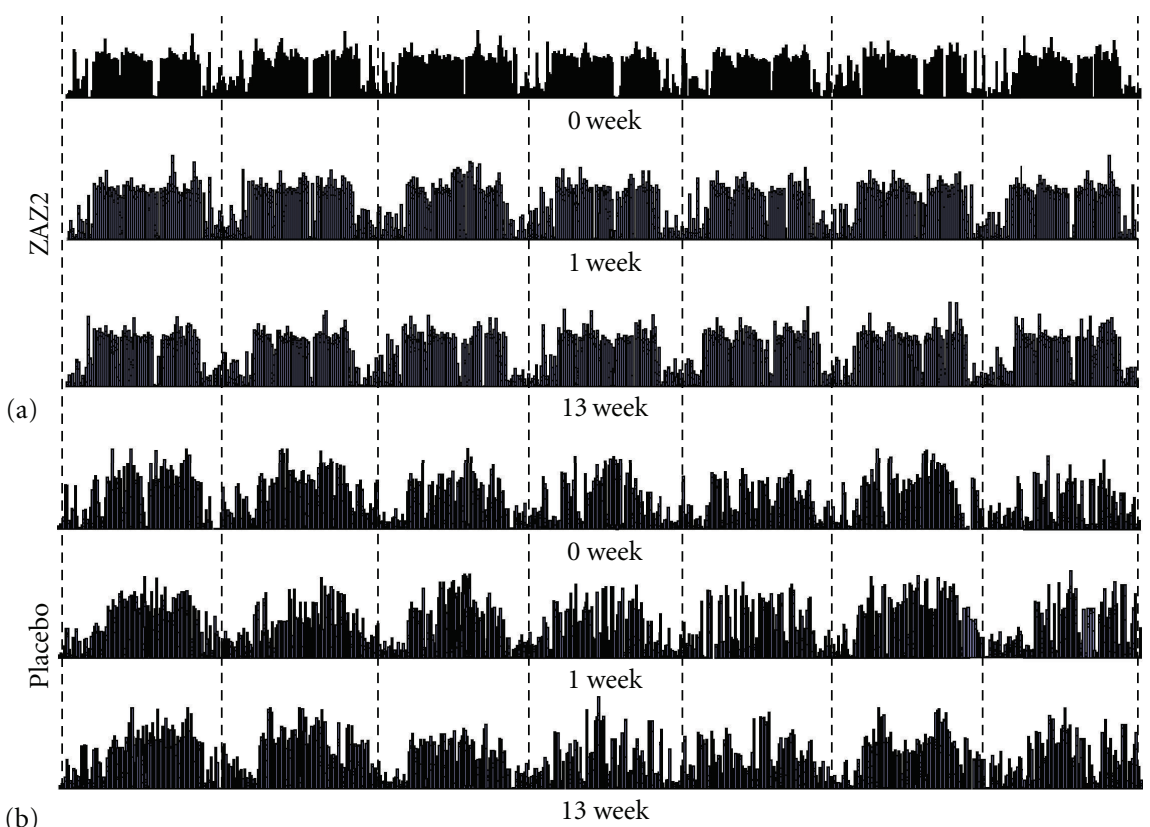

FIGURE 1: Daily profiles of AMI counts demonstrated the biological rhythm after granule ingestion in the ZAZ2 group (a) and in the placebo group (b). Each dash in the recordings represents midnight.

of "switching" patterns between high and low values in a statistical sense. The average wavelet coefficients exhibited linear relationships in the range of scales from $8 \mathrm{~min}$ to $35 \mathrm{~min}$ both for the ZAZ2 and placebo groups (Figure 2(b)). The local power-law exponent $\alpha$ values during both awake time and sleep time were significantly decreased both 1 week and 13 weeks after taking ZAZ2, but not after taking placebo granule (Table 2 and Figure $2(\mathrm{c}), P<.01$; Bonferroni test).

As the exploratory outcome of this study, most of the secondary symptoms were improved by the ZAZ2 treatment, whereas only a few symptoms in the placebo group were transiently improved in week 1 (Table 3 ).

\section{Discussion}

In this study, we demonstrate that ZAZ2, a TCM, ameliorates the disability of PD patients using the analysis of power-law temporal autocorrelation of the AMI records together with conventional UPDRS. Because a recent study indicated that improvement of the scores in UPDRS Part II reflect the longterm outcome of the patients [21], improvements of scores in UPDRS Part II, II + III, and IV likely reflect the beneficial effects of ZAZ2 on the patients' overall ADL as an endpoint of the treatment. ZAZ2 induced no significant adverse effects and was tolerable by more than $98 \%$ of the participants.

We previously demonstrated that the change in the local power-law exponent $\alpha$ is a quantitative predictor for evaluating the akinesia changes in $\operatorname{PD}[15,20]$. Because the $\alpha$-values indicate persistency, lower $\alpha$-values correspond to more frequent switching of behavior or higher physical activity $[15,20]$. Therefore, the significant reduction in the $\alpha$-values after ZAZ2 likely represents the improvement of motor function of the patients as a whole, which is in accordance with the improvement of scores of UPDRS Part II and Part II + Part III.

The effects of ZAZ2 are also demonstrated by the improvement in the scores for the secondary symptoms (Tables 2 and 3 ) and in the wake-sleep cycle (Figure 1) as 


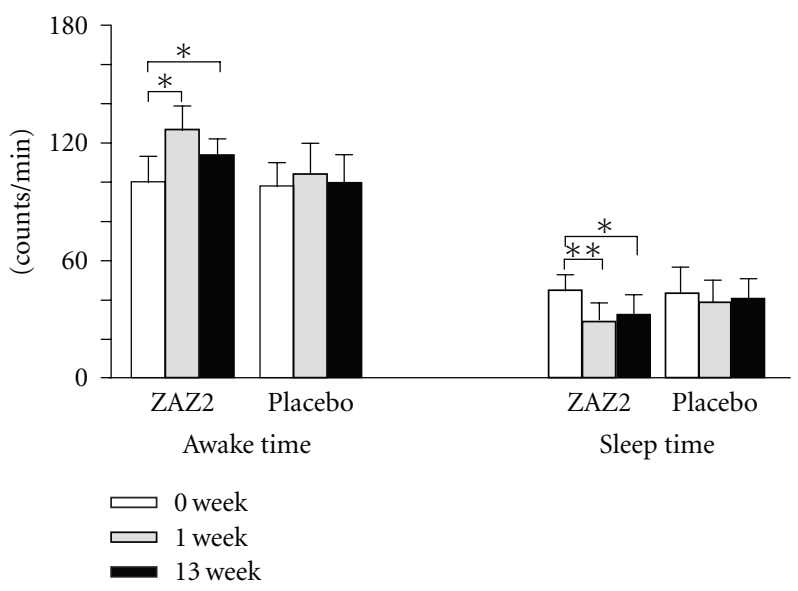

(a)

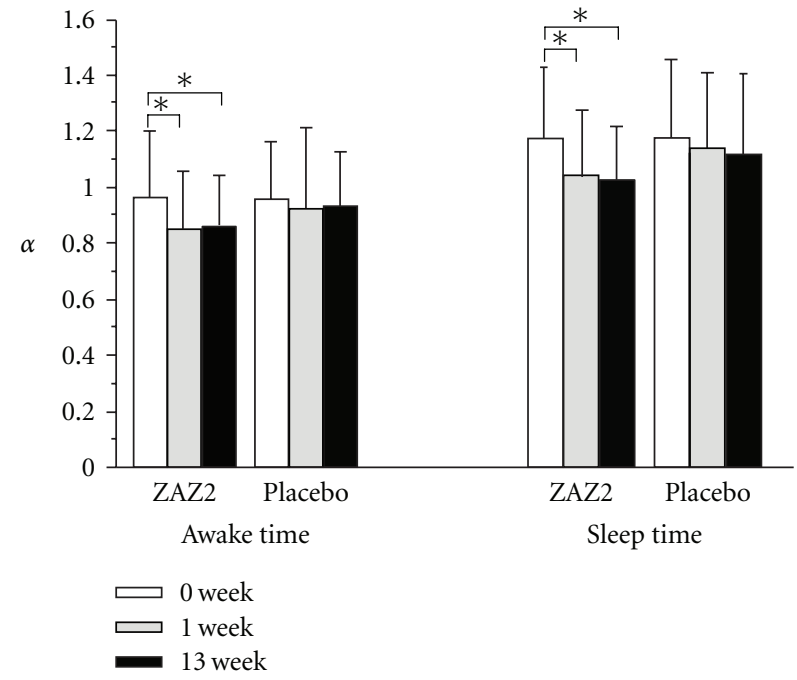

(c)

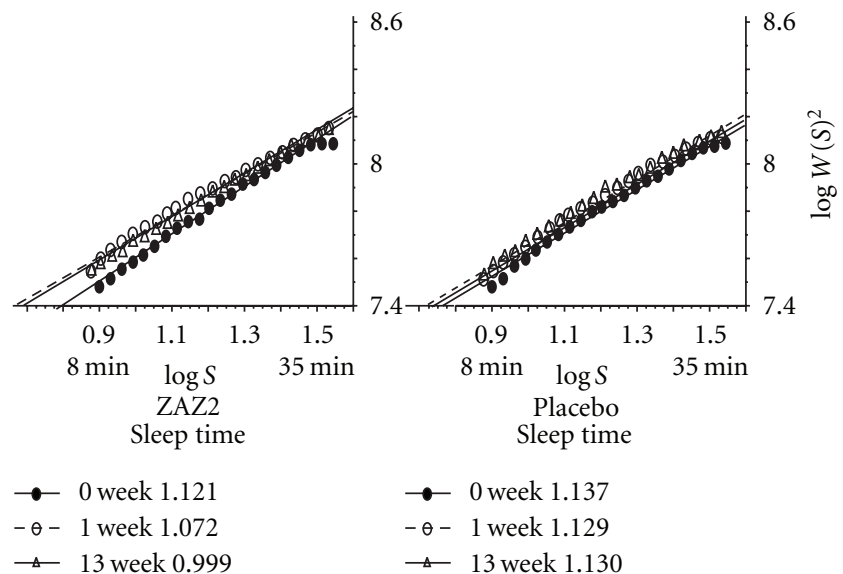

b)

FIgURE 2: Before and after administration of granules of ZAZ2 and placebo for mean counts of physical activity (a), average wavelet coefficients, as a function of the wavelet scale for awake time and sleep-time, the slopes are power-law exponents, $\alpha$ (b), and comparisons of the mean $\alpha$ (c).

exploratory outcomes of this study. Indeed, activity during sleep time was markedly decreased and the local power-law exponent $\alpha$ was significantly decreased during sleep time as well as during awake time (Figures $2(\mathrm{a})$ and 2(c)). Because sleep disturbance, which is frequent among patients with $\mathrm{PD}$, is thought to be due to disruption of the nighttime effects of levodopa [22], improvement of wake-sleep rhythm is likely a reflection of an improvement in parkinsonism. ZAZ2 may have beneficial effects on ADL by ameliorating the symptoms resulting from parkinsonism without exacerbating the L-dopa-induced adverse effects, as demonstrated in the improvement in scores of UPDRS Part IV.

TCM ameliorates various symptoms, particularly the ageing-related symptoms that are called shen $x u$ (kidney deficiency) in Chinese $[10,11]$. Shen (the kidney) denotes a functional visceral system (zang) that plays a central role in the regulation of growth, maturation, and ageing, and is subdivided into shen yang (kidney yang) and shen yin (kidney yin). Kidney yang can be described as the driving forces of all metabolic processes that improve the movements of the body. The production of kidney yin is considered to be effective at increasing nutrition to the muscles and improving the smoothness of the movements of the body by constituting the structive potential for the production of kidney yang. Based on this concept, TCM aims to potentiate a diminishing vitality of this transformative cycle caused by a decline of the essence (jing), which is stored in the kidneys and underpins the functions of both kidney yin and yang $[23,24]$.

Among the 14 components of ZAZ2, Morindae officinalis radix and Desertliving Cistanche might strengthen the "kidney yang" while Asnaragus cochinchinensic, Cornus officinalis, and Rehmanniae radix might increase the "kidney yin." The other components ameliorate secondary symptoms such as 
headache, vertigo, and tinnitus (Uncaria rhynchophylla and Rhizoma gastrodiae), enhance the strength of the "kidney" (Paeonia lactiflora, Puerariae radix, Salviae Miltiorrhizae radix, Curcuma longa Linn, and Rhizoma chuanxiong), or increase blood circulation in the brain (Arisaema consanguineum Schott and Acorus tatarinowii). In addition, the inhibitory effects of TCM on fibril formation and inhibited $\mathrm{A} \beta$ aggregation (Uncaria rhynchophylla) [25], and on apoptosis and the neurobehavioral impairment of 1methyl-4-phenylpyridinium ion $\left(\mathrm{MPP}^{+}\right)$-induced $\mathrm{PD}$ model mice (desertliving Cistanche) [26, 27] and rats (Uncaria rhynchophylla, Cornus officinalis, Rehmanniae radix, and Paeonia lactiflora) [28] have been reported. Whether the herbs in ZAZ2 contain L-dopa or anticholinergic agents has not been demonstrated. Therefore, ZAZ2 may be potentially effective in the regulation of motor and various nonmotor symptoms of patients with PD without inducing the adverse effects of conventional anti-parkinsonism drugs. ZAZ2 is tolerable for long-term administration, and hence is likely a suitable choice as an additional drug for long-term control of the symptoms of PD.

\section{Authors' Contribution}

Weidong Pan participated in the entire study, formulated the study concept and design, provided statistical expertise, and assisted with drafting of the manuscript; Shin Kwak participated in the entire study and assisted with concept and design, and drafting of the manuscript; Yun Liu participated in part of content and data compilation; Yan Sun participated in part of content and data compilation; Zhenglong Fang participated in part of content and data compilation; Baofeng Qin participated in part of content and data compilation; Yoshiharu Yamamoto participated in part of content and critical reversion of the manuscript for important intellectual content.

\section{Disclosure}

All authors have no stock ownership in medically related fields, no consultancies, no advisory Boards, no partnerships, no grants, no intellectual property rights, no expert testimony, no employment, or no contracts as well as no royalties.

\section{Conflict of Interests}

The authors declared that there is no conflict of interest.

\section{Acknowledgments}

The authors express their appreciation for patience and dedication of clinical research and hospital staff involved in this clinical study and to the subjects and their families. This work was sponsored by Shanghai Pujiang Program from Science and Technology Commission of Shanghai Municipality (no. 09PJ1409300).

\section{References}

[1] R. Pahwa, S. A. Factor, K. E. Lyons et al., "Practice parameter: treatment of parkinson disease with motor fluctuations and dyskinesia (an evidence-based review): report of the quality standards subcommittee of the american academy of neurology," Neurology, vol. 66, no. 7, pp. 983-995, 2006.

[2] D. E. Hobson, A. E. Lang, W. R. Martin, A. Razmy, J. Rivest, and J. Fleming, "Excessive daytime sleepiness and suddenonset sleep in Parkinson disease: a survey by the Canadian movement disorders group," Journal of the American Medical Association, vol. 287, no. 4, pp. 455-463, 2002.

[3] C. L. Comella, "Daytime sleepiness, agonist therapy, and driving in Parkinson disease," Journal of the American Medical Association, vol. 287, no. 4, pp. 509-511, 2002.

[4] I. Arnulf, E. Konofal, M. Merino-Andreu et al., "Parkinson's disease and sleepiness: an integral part of PD," Neurology, vol. 58, no. 7, pp. 1019-1024, 2002.

[5] W. G. Ondo, K. Dat Vuong, H. Khan, F. Atassi, C. Kwak, and J. Jankovic, "Daytime sleepiness and other sleep disorders in Parkinson's disease," Neurology, vol. 57, no. 8, pp. 1392-1396, 2001.

[6] J. M. Miyasaki, K. Shannon, V. Voon et al., "Practice parameter: evaluation and treatment of depression, psychosis, and dementia in Parkinson disease (an evidence-based review): report of the quality standards subcommittee of the American Academy of Neurology," Neurology, vol. 66, no. 7, pp. 9961002, 2006.

[7] K. R. Chaudhuri and A. H. Schapira, "Non-motor symptoms of Parkinson's disease: dopaminergic pathophysiology and treatment," The Lancet Neurology, vol. 8, no. 5, pp. 464-474, 2009.

[8] A. Park and M. Stacy, "Non-motor symptoms in Parkinson's disease," Journal of Neurology, vol. 256, no. 3, pp. 293-296, 2009.

[9] P. A. G. M. De Smet, "Herbal remedies," The New England Journal of Medicine, vol. 347, no. 25, pp. 2046-2056, 2002.

[10] J. K. Chen, "Menopause: western and traditional Chinese medicine perspectives-part II," Acupuncture Today, vol. 3, no. $5,2002$.

[11] G. Maciocia, Obstetrics and Gynaecology in Chinese Medicine, Churchill Livingstone, Edinburgh, UK, 1998.

[12] H. Zhao, W. W. Li, and J. P. Gao, "Clinical trial on treatment of Parkinson's disease of Gan-Shen yin deficiency type by recipe for nourishing Gan-Shen," Zhongguo Zhong Xi Yi Jie He Za Zhi, vol. 27, no. 9, pp. 780-784, 2007.

[13] X. F. Lian and X. D. Luo, "Effect of TCM treatment according to syndrome differentiation in enhancing curative effect and reducing side-effect of madopa," Zhongguo Zhong Xi Yi Jie He Za Zhi, vol. 27, no. 9, pp. 796-799, 2007.

[14] P. Martinez-Martin, A. Gil-Nagel, L. M. Gracia, J. P. Gomez, J. Martinez-Sarries, and F. Bermejo, "Unified Parkinson's disease rating scale characteristics and structure. The Cooperative Multicentric Group," Movement Disorders, vol. 9, no. 1, pp. 76-83, 1994.

[15] W. Pan, K. Ohashi, Y. Yamamoto, and S. Kwak, "Powerlaw temporal autocorrelation of activity reflects severity of parkinsonism," Movement Disorders, vol. 22, no. 9, pp. 13081313, 2007.

[16] K. Ohashi, L. A. N. Amaral, B. H. Natelson, and Y. Yamamoto, "Asymmetrical singularities in real-world signals," Physical Review E, vol. 68, no. 6, Article ID 065204, 4 pages, 2003. 
[17] M. H. Teicher, "Actigraphy and motion analysis: new tools for psychiatry," Harvard Review of Psychiatry, vol. 3, no. 1, pp. 1835, 1995.

[18] Medicine GMAoTC, "Definition and evaluation of parkinsonism in TCM," Journal of Beijing University of Traditional Chinese Medicine, vol. 14, pp. 39-41, 1992.

[19] A. J. Hughes, S. E. Daniel, L. Kilford, and A. J. Lees, "Accuracy of clinical diagnosis of idiopathic Parkinson's disease: a clinico-pathological study of 100 cases," Journal of Neurology Neurosurgery and Psychiatry, vol. 55, no. 3, pp. 181-184, 1992.

[20] W. Pan, R. Soma, S. Kwak, and Y. Yamamoto, "Improvement of motor functions by noisy vestibular stimulation in central neurodegenerative disorders," Journal of Neurology, vol. 255, no. 11, pp. 1657-1661, 2008.

[21] M. B. Harrison, S. A. Wylie, R. C. Frysinger et al., "UPDRS activity of daily living score as a marker of Parkinson's disease progression," Movement Disorders, vol. 24, no. 2, pp. 224-230, 2009.

[22] K. Suzuki, Y. Okuma, N. Hattori et al., "Characteristics of sleep disturbances in Japanese patients with Parkinson's disease. A study using Parkinson's disease sleep scale," Movement Disorders, vol. 22, no. 9, pp. 1245-1251, 2007.

[23] V. Scheid, "Traditional chinese medicine-what are we investigating? The case of menopause," Complementary Therapies in Medicine, vol. 15, no. 1, pp. 54-68, 2007.

[24] M. Porkert, The Theoretical Foundations of Chinese Medicine: Systems of Correspondence, MIT Press, Cambridge, UK, 1973.

[25] H. Fujiwara, K. Iwasaki, K. Furukawa et al., "Uncaria rhynchophylla, a Chinese medicinal herb, has potent antiaggregation effects on Alzheimer's beta-amyloid proteins," Journal of Neuroscience Research, vol. 84, no. 2, pp. 427-433, 2006.

[26] W. W. Li, R. Yang, and D. F. Cai, "Protective effects of Cistanche total glycosides on dopaminergic neuron in substantia nigra of model mice of Parkinson's disease," Zhongguo Zhong Xi Yi Jie He Za Zhi, vol. 28, no. 3, pp. 248-251, 2008.

[27] X. Pu, X. Li, H. Li, P. Tu, Z. Song, and C. Li, "Campneoside II of Cistanche tubulosa (Schenk) R. Wight protects neurons from apoptosis induced by neurotoxin 1-methyl4-phenylpyridinium(MPP+)," Journal of Peking University (Health Sciences), vol. 33, pp. 217-220, 2001.

[28] W. Li, D. Cai, and X. Chen, "Effects of yanggan xifeng recipe on changes of neurological behavior of rat of Parkinson's model," Zhongguo Zhong Xi Yi Jie He Za Zhi, vol. 20, no. 12, pp. 920-922, 2000. 


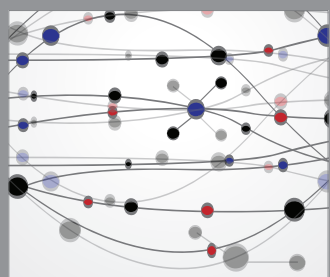

The Scientific World Journal
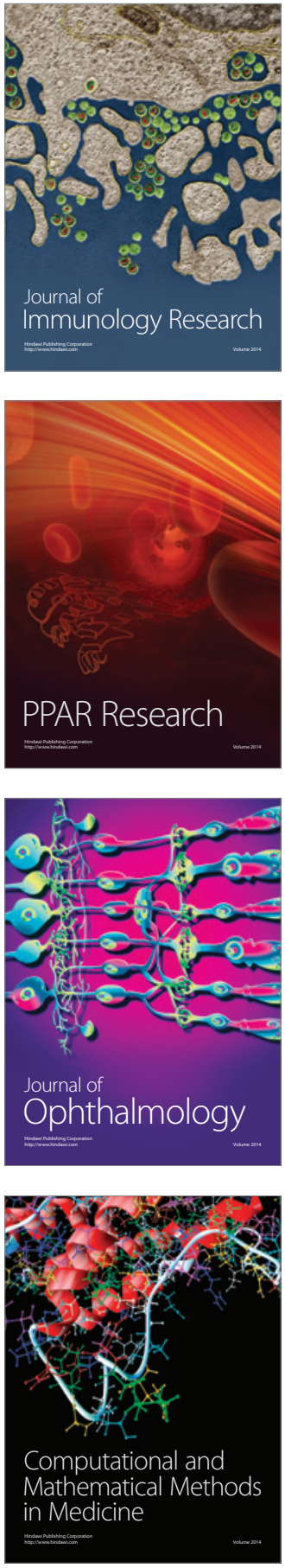

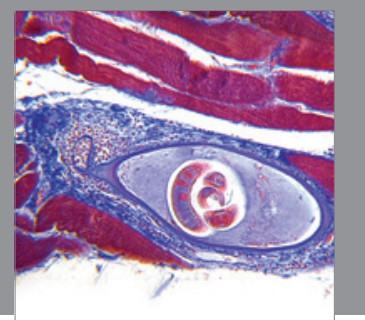

Gastroenterology

Research and Practice
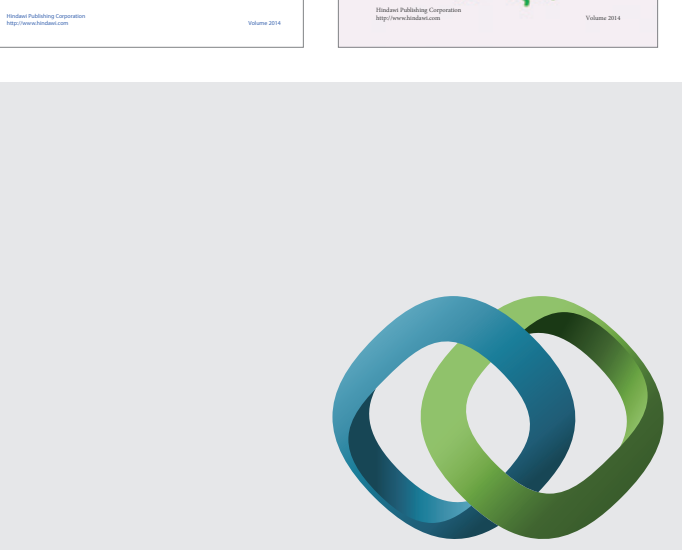

\section{Hindawi}

Submit your manuscripts at

http://www.hindawi.com
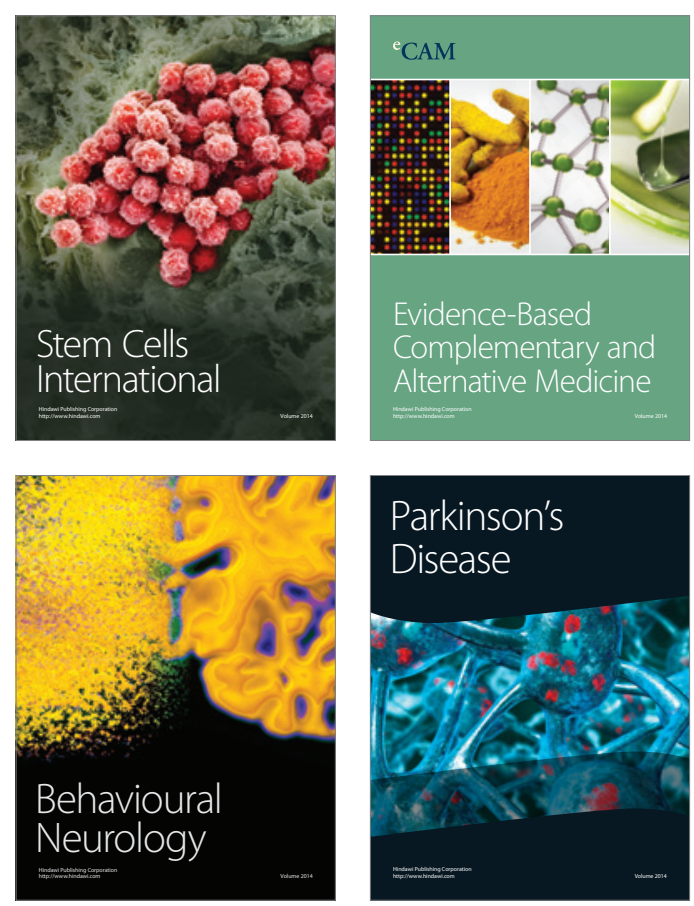

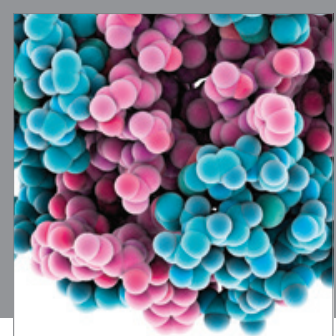

Journal of
Diabetes Research

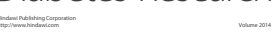

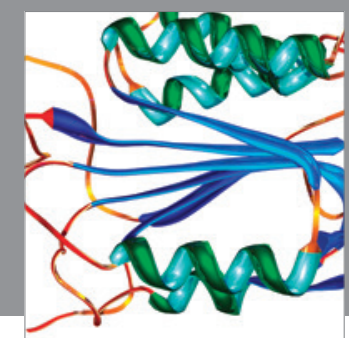

Disease Markers
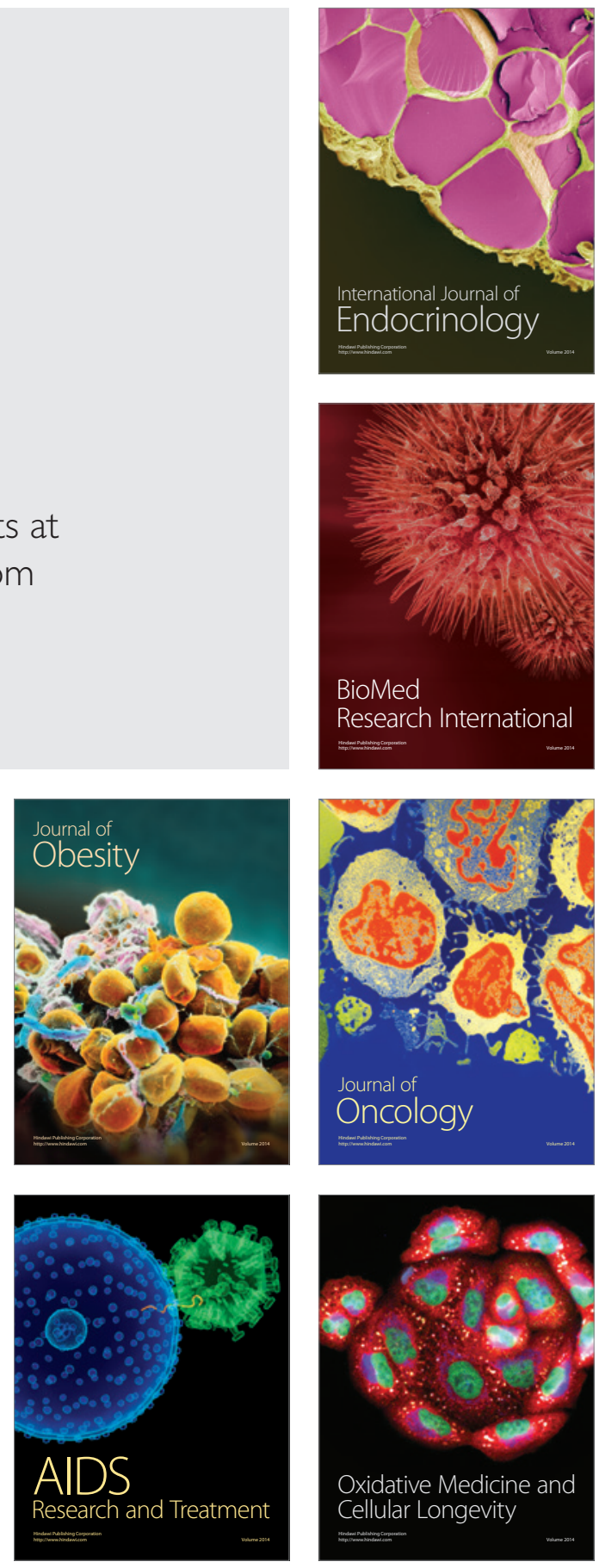